ethnography approach. Santuario El Cañi, Chile.

\author{
MARTín FonCK ${ }^{* *}$ \\ DANIELA JACOB ${ }^{* * *}$
}

\section{"Escuchando el llamado del bosque": explorando las dimensiones afectivas de la conservación ambiental desde la etnografía multiespecies. Santuario El Cañi, Chile}

"Listening to the forest's call" exploring the affective dimensions of environmental conservation through a multiespecies

\footnotetext{
Se le agradece al Dr. Piergiorgio Di Giminiani en marco de cuyo proyecto esta investigación fue realizada, y sin cuyos valiosos comentarios no se hubiera llegado a la versión final de este artículo. Este proyecto fue financiado por Centro de Estudios Interculturales e Indígenas, CIIR (CONICYT/ FONDAP/15110006). A su vez se le agradece a los guías locales del Santuario El Cañi, Roberto y Manuel, por su apoyo y entusiasmo, así como al resto de la comunidad local de Pichares. Agradecemos a Rod por sus enseñanzas en el bosque. También a Tomás Ibarra, Tomás Altamirano y su equipo de trabajo por permitirnos participar en su valioso proyecto de investigación.

** Candidato a doctor en Antropología Social, Ludwig-MaximiliansUniversität München y Rachel Carson Center for Environment and Society (LMU). Leopoldstr. 11a 80802 Munich. E-mail: martin. fonck@rcc.Imu.de

*** Candidata a Magíster en Antropología Social, London School of Economics and Political Science, Houghton St, London WC2A 2AE. E-mail: d.p.jacob-pinto@Ise.ac.uk
}

\section{Resumen}

En el pasado se ha cuestionado el rol social de los proyectos de conservación medioambiental, debido a la falta de participación de las comunidades locales. Actualmente, esto se ha reformulado, pasando a plantearse la participación de las comunidades locales como desafío principal de los proyectos de conservación. En este artículo se plantea que esta reformulación debe ir acompañada a su vez de una comprensión más amplia, abordando las relaciones entre especies como parte fundamental para el análisis y la discusión sobre la conservación medioambiental. Tomando como base dos años de trabajo etnográfico en la zona de Pichares, novena región de Chile, en la que se ubica el Santuario de la Naturaleza El Cañi, se ha dado cuenta de cómo el rol social de la conservación está supeditado a las formas de convivencia con otras especies (aves, vacas y árboles). Específicamente, la tensión existente entre los ambientalistas y los campesinos locales debe ser abordada desde las prácticas de vida en el bosque y los desafíos que la interconexión entre especies plantea a la conservación. Por otra parte, es fundamental para comprender el desarrollo de la colaboración local, el rol que juegan los guías locales ostentando valores y prácticas tanto campesinas como ambientalistas. Entre estas 
se ha cultivado la afectividad en las relaciones con ciertas especies de árboles, insectos y aves, que desvelan una multiplicidad de relaciones de afecto entre especies que llaman a que el bosque sea cuidado.

Palabras Clave: Conservación, etnografía multiespecies, comunidad local, afectos, bosque

\section{Abstract}

In the past, the social role of environmental conservation has been put into question due to the lack of participation of the local communities. Actually, this has been reformulated, being the participation of the local communities the main focus of the conservation projects. In this article it is stated that this reformulation must be accompanied by a broader comprehension, addressing the relations between different species as a fundamental part of the analysis and discussion of environmental conservation. Based on two years of ethnographic research in the area of Pichares, ninth region of Chile, where the El Cañi Nature Sanctuary is located, it has been analysed how the social role of conservation is subordinated to the forms of coexistence with other species (birds, cows and trees). Specifically, the tension between environmentalists and local peasants must be addressed from life practices in the forest and the challenges that the interconnection between species poses to conservation. On the other hand, it is fundamental to understand the development of local collaboration, through the role played by local guides that share values and practices with both peasants and environmentalists. Among these, affectivity has been cultivated in human relationships with certain species of trees, insects and birds. These reveal a multiplicity of relationships of affection between species that call for the forest to be taken care of.

Key words: Conservation, multispecies ethnography, local community, forest

\section{Introducción}

Hoy en día existen más de 300 áreas protegidas privadas en el sur de Chile, las que cubren 1.604.000 hectáreas, siendo aproximadamente el 2,12\% del territorio nacional (Ogden y Holmes, 2015). Estas vienen a complementar la labor de conservación estatal, efectuada por medio del Sistema Nacional de Áreas Protegidas del Estado (SNASPE) que corresponde al 19\% del territorio de Chile Continental (JorqueraJaramillo et al., 2012). Las áreas de protección privadas se establecen desde ciertos grupos de la sociedad civil —filántropos conservacionistas nacionales e internacionales, grandes ONGs conservacionistas y ONGs nacionales, activistas ambientales, grupos locales de conservación, organizaciones de base, entre otros-- los cuales reaccionan frente a la opción histórica tomada por el Estado Chileno de priorizar la extracción de recursos naturales, por sobre la generación de políticas de conservación, manejo y restauración del bosque nativo en el largo plazo (Klubock, 2014) ${ }^{1}$.

En relación al rol social de la conservación, privada y estatal, una de las críticas fundamentales ha girado en torno a la prohibición del uso de

Si bien, esta ha sido el enfoque prioritario es necesario reconocer los tempranos esfuerzos en conservación medioambiental en Chile, como por ejemplo la aprobación en 1907 de la Reserva Forestal Malleco (Camus y Lazo, 2014) 
recursos naturales tradicionalmente empleados por poblaciones vecinas a las áreas protegidas (Brockington, 2002; Catalán, 2015; Catalán et al. 2006). En la actualidad, se ha llegado a consenso en la necesidad de involucrar a las comunidades locales, sus propios intereses y conocimientos para que el desarrollo de la conservación sea sustentable en el tiempo. Esto pues la conservación ambiental se ha practicado desde la instauración de reservas naturales como un acto de custodia de ambientes prístinos para ser protegidos de la invasión humana (Campbell, 2005; Cronon, 1996). Para solucionar este problema se han desarrollado programas y políticas inspirados en los principios de la conservación basada en la comunidad (Brosius et al., 1998). Estas iniciativas buscan enlazar la conservación de la biodiversidad con la generación de ingresos económicos para la comunidad local a partir de la conservación de un lugar (Vargas \& Díaz, 2014).

A pesar de los beneficios que pueda traer aparejada la conservación basada en la comunidad, sostendremos que la definición que este tipo de iniciativas hacen de la comunidad local, entendiéndose como conformada sólo por seres humanos, es un reducción que simplifica toda la complejidad asociada a las prácticas cotidianas en estas áreas. Esto pues este tipo de iniciativas siguen operando sobre la distinción naturaleza-cultura (Descola, 2013), la cual ha sido fundamental para el pensamiento y práctica ambiental occidental (Lorimer, 2012). Esta separación entre naturaleza y cultura por un lado reproduce la idea de naturaleza como algo que tiene una realidad única y esencial, y por otro lado presupone que el ser humano ocupa un lugar especial en el cosmos, escindido de esta naturaleza (Stengers, 2010). Siguiendo a Latimer \& Miele (2013) planteamos que esta separación no sólo genera pobreza epistemológica, sino que también ayuda a perpetuar una actitud sobre lo no humano que lo observa como un recurso que el ser humano puede emplear a su antojo. Planteamos que en el contexto actual de cambio climático y degradación medioambiental, caracterizado por la influencia humana en los procesos geológicos de la tierra (Crutzen, 2002) se vuelve necesario desmontar este tipo de aparatajes conceptuales en pos de generar otras formas de relación con lo no humano, distintas a las que han llevado a la situación actual de crisis.

En este contexto, pensadores como Latour (2004) plantean que la distinción naturaleza cultura es sólo analítica, existiendo fácticamente hibridajes entre elementos que son considerados como pertenecientes a estas dos categorías. Estos elementos formarían ensamblajes conectados en red; estando la agencia distribuida tanto en seres humanos como no humanos. De este modo Lorimer (2012) considera la existencia de múltiples naturalezas que son constituidas relacionalmente (Lorimer, 2012) desensencializando la naturaleza como algo unívoco y definido por oposición a la esfera de la humano. En esta línea, Donna Haraway (2008) rescata los procesos de mutua afectación y co constitución que suceden entre distintas especies, acuñando el término de especies compañeras. Las identidades, y afinidades, de las especies compañeras emergen de las relaciones que se establecen entre ellas, y por lo tanto ninguna de estas puede entenderse como aislada. En este sentido, los humanos y no humanos "nunca son puramente sí mismos" (Haraway, 2008:250), llegando a plantearse que la "naturaleza humana" es una relación interespecies (Tsing, 2012). Haraway (2008) plantea que esta perspectiva viene a disputar 
las prácticas de conocimiento dominantes, ya que este estaría implicado también en los procesos de producir conocimiento, siendo el científico afectado por su supuesto objeto de estudio (Latimer \& Miele, 2013).

Para propósitos de este artículo rescataremos el concepto de afecto como parte constitutiva de las relaciones entre distintas especies. La idea del afecto fue acuñada por Baruch Spinoza quien afirmó que el afecto implica "afectar y ser afectado" (Massumi, 2015; Gregg \& Seigworth, 2009). Si bien el afecto como concepto tradicionalmente ha sido asociado a las emociones, la presente definición pone en relevancia el potencial del afecto para pensar más allá de la esfera humana. El afecto implicaría una comprensión del mundo como un proceso de transformación perpetua, en que los seres se involucran mutuamente desde sus cuerpos (Massumi, 2015). El afecto de este modo pone en centro la mutua involucración en la que los humanos habitamos con otras especies, involucración que no es sólo simbólica, sino también material. En este sentido, el afecto como enfoque propone comprender las relaciones entre especies principalmente desde su carácter encorporalizado. El cuerpo es la interface por medio del cual tenemos la capacidad de volvernos sensibles a las múltiples interacciones que permiten la vida en la tierra. Encarnandose en interacciones específicas, el afecto depende de cada cuerpo, para los efectos que produce. En esta línea Navaro Yashin (2012) plantea que distintas entidades transmiten afecto de acuerdo a quienes los experimentan y sus propios conocimientos previos. De esto puede desprenderse que la capacidad de los humanos para dejarse afectar por ciertos seres no es la misma, estando mediada por los saberes respectivos que tienen los sujetos. En este contexto Kirksey \& Helmreich (2010) han acuñado el término de etnografía multiespecies, la cual aborda etnográficamente la relacionalidad entre seres humanos y no humanos. Esta aproximación, aliada a proyectos como la 'antropología de la vida' desarrollada por Kohn (2013), aborda las relaciones entre seres de distintas especies y sus dinámicas conjuntas. Desde esta perspectiva hemos analizado los conflictos y prácticas presentes en el Santuario El Cañi profundizando en la complejidad de relaciones en torno a la conservación.

\section{Métodos}

Los resultados de esta investigación fueron obtenidos tras dos años de trabajo de campo etnográfico en la zona de Pichares, novena región de Chile, entre los años 2014 y 2015. El trabajo etnográfico se compone de la práctica de la observación participante en terreno, en la que se convive de cerca con los actores involucrados, participando de sus actividades cotidianas. De este modo se tuvo acceso al como estos producen sentido de su medioambiente, y al mismo tiempo a las redes de relaciones ecológicas en las que estos se ven involucrados. Cabe destacar como herramienta fundamental la realización de entrevistas caminando con los actores por el santuario de conservación basadas en metodologías móviles de observación participante (Evans y Jones, 2011; Kusenbach, 2003). Durante el proceso de investigación los recorridos por el santuario con campesinos, ambientalistas y guías, fueron una herramienta significativa de análisis ya que permitieron generar una aproximación a la vida de las especies no humanas en El Cañi y a las 
interacciones entre estas. Particularmente la participación en la restauración anual de los cercos, nos permitió introducirnos en las tensiones con los bovinos (bos primigenius taurus), observando sus huellas y rutas por el bosque, a la vez que encontrarnos directamente con ellos en el bosque. En este sentido, los recorridos observados de estos animales permitieron acompañar los discursos y valoraciones de los humanos, con las interacciones cotidianas entre especies que se dan en el parque mismo. A su vez, se realizaron 54 entrevistas semi estructuradas a actores relevantes vinculados a el Santuario El Cañi. De estos casos, 20 entrevistas fueron realizadas en predios campesinos en los alrededores del santuario, donde se logró realizar una aproximación a la valoración y rol cotidiano del ganado de parte de la comunidad local, entre otras valoraciones ambientales. También se realizaron talleres de conversación en torno a la historia del proyecto y sus implicancias ecológicas involucrando campesinos, ambientalistas y guías locales.

Para llevar cabo una aproximación etnográfica multiespecies dentro del espacio del Santuario El Cañi, se ha considerado la movilidad en el bosque como una clave metodológica a seguir. Basándonos en los planteamientos de Ingold (1995) proponemos que el espacio es construido por las distintas especies que lo habitan mediante sus propios procesos de vida, los que incluyen alimentación y desplazamiento. Al mismo tiempo, se escogió poner atención a cómo el medio ambiente es producido materialmente mediante prácticas humanas (Robbins, 2004). De este modo se realizaron observaciones en torno a los cercos y senderos propuestos por los miembros del proyecto de conservación.

\section{El Santuario El Cañi:}

Ubicado en la localidad de Pichares, comuna de Pucón, Región de la Araucanía, el Santuario El Cañi es uno de los primeros proyectos de conservación privada en Chile. Tiene una extensión de 400 hectáreas ocupando el cerro Cañi, que tiene una altura de 1525 metros. Se caracteriza por la presencia de bosque templado andino, albergando especies como Coihue (Nothofagus dombeyi), Lenga (Nothofagus pumilio) y Araucaria (Araucaria Araucana). El bosque que forma parte del proyecto de conservación se encuentra en los sectores altos de la cordillera, sobre 700 metros de altura, como en la gran parte de las áreas de conservación público y privada en Chile (Ibarra et. al., 2012). Las personas que viven en torno a esta área protegida se concentran en la localidad de Pichares. En este sector los habitantes se autoidentifican principalmente como campesinos y se dedican a actividades ganaderas y forestales. Bajo este contexto, las principales amenazas que afectan al bosque es la fragmentación fruto de la actividad humana tales como la explotación del bosque nativo, la plantación de especies exóticas y la agricultura. Sin embargo, en los últimos años un factor de relevancia ha sido el incremento del turismo en el sector y la migración por estilo de vida de personas desde sectores urbanos asociados al ecoturismo y proyectos ambientales (Hidalgo y Zunino, 2011). Por todo lo anterior se trata de un paisaje con patrones espaciales en transformación fruto de actividades económicas y nuevas interacciones sociales con el medioambiente (Petitpas et. al., 2016).

La historia de el Santuario El Cañi se remonta al año 1989, cuando la empresa forestal neozelandesa Carter Holt Harvey se interesa en 
comprar el terreno. Una pareja de extranjeros, quienes habitaban en las cercanías del lugar, se entera de la situación y toma acciones para interponerse ante esto, con el propósito de proteger al bosque nativo que se encontraba en el Cerro El Cañi. Se contactan con ambientalistas estadounidenses vinculados la ONG Ancient Forest International (AFI), para ayudar a recaudar fondos para efectuar la compra. De este modo se recurre a la Frank Weeden Foundation, quienes aportan US\$50,000, a los que se le suma posteriormente aportes de privados y empresas como Patagonia, que aporta US\$50,000, y Esprit-Chile, que aporta US $\$ 40,000$, ambas asociadas a la pareja de ambientalistas estadounidenses Kristine y Douglas Tompkins (Scott, 2002). De hecho, en la misma época esta pareja se encontraba involucrada en el proceso de compra del controversial Parque Pumalín, de una extensión de 325,000 hectáreas, el cual por su tamaño y posición parecía amenazar la soberanía nacional (Jones, 2012). En el año 1992, tras reunir US\$160,000 se concretó la compra del terreno que había sido temporalmente adquirido por la pareja de extranjeros, poniéndolo a nombre de la recientemente creada Fundación Lahuén.

El año 1994, el Santuario el Cañi toma un nuevo camino. Tomando críticas realizadas a modelos de conservación anteriores, se comienza a destacar la importancia del involucramiento de la comunidad local aledaña al área conservada, planteando la educación ambiental como una forma de integración. De este modo se genera una asociación con CEAL (Centro de Educación al Aire Libre), iniciativa del británico Rod Walker, para hacer capacitaciones a habitantes de la zona. A esto se suma la necesidad económica, ya que el proyecto comenzaba a quedarse sin fondos, buscando promover nuevas fuentes de ingreso para su mantención, por lo que el año 1997, se construye un vivero de árboles nativos, con el apoyo de un fondo público, el cual genera interés y participación de parte de la comunidad local. El año 1998 los fondos internacionales se acaban definitivamente, por lo que se crea la organización comunitaria "Grupo Guías del Cañi", bajo el alero de la municipalidad. Entre los fines que se planteó la agrupación destacan la administración de la reserva, la continuación de los proyectos de la fundación, el ecoturismo y la educación ambiental. El año 1999, en un hecho sin precedentes, se le traspasa la administración a esta agrupación, quedando como administradores los guías locales Manuel Venegas y Roberto Sanhuesa. Estos comienzan el camino para abrir el parque al público, dando paso desde el proyecto de conservación original, cerrado y receloso de los visitantes, hacia la educación ambiental de estos, en pos de generar un proyecto sustentable en el tiempo. De este modo se empieza a cobrar entrada al público visitante, ofreciendo también visitas guiadas por un monto adicional (Scott, 2002).

\section{4. "Las vacas siempre han subido el cerro": Prácticas de vida campesina en torno al Santuario el Cañi.}

La población campesina de Pichares se encuentra integrada por comunidades mapuche, y por comunidades chilenas colonas a la zona, llegadas tras la colonización del Estado chileno del territorio histórico de este pueblo indígena (Bengoa, 2002). La vida campesina sucede un poco de espaldas al santuario El Cañi, si bien este cobra importancia en ciertas épocas del año. Nuestras interacciones y conversaciones con los vecinos en torno a El Cañi se dieron 
por lo general en el interior de los hogares o en las inmediaciones de sus casas, o en los potreros donde estos guardaban a las vacas. En las conversaciones que tuvimos, nos fue posible notar cómo la forma de vida campesina se encuentra relacionada económica y culturalmente a la vida de las vacas. Tradicionalmente, los habitantes de Pichares y Coilaco han tenido economías en que dependen de la carne de vaca, tanto para el propio consumo, como para su venta. Esto puede observarse en ciertos detalles, como el hecho de que tradicionalmente el término kulliñ significa tanto ganado como dinero en mapudungun. En este sentido, la vaca es una especie que condiciona las actividades y los usos tanto de los predios rurales como del bosque próximo. Miguel, campesino de Pichares, nos comentaba que las vacas "siempre han subido el cerro" para alimentarse en verano, manifestándonos que estas prácticas que eran comunes antes de la llegada de los ambientalistas, y que se han visto entorpecidas desde la llegada de estos. Tradicionalmente, esta práctica tiene el nombre de veranada, siendo crucial para la economía de los pequeños propietarios, quienes de tal manera utilizan pastos silvestres en las zonas altas de la montaña y permiten que sus propios pastos descansen en verano.

De hecho, en la práctica de la veranada, cuando los campesinos suben al cerro El Cañi a buscar sus vacas, en general lo hacen siguiendo sus huellas. Estando la relación con el cerro mediada por estas, y no por los senderos que han ido elaborando los ambientalistas con el tiempo. Las vacas, quiénes tienden a transitar siempre por los mismos lugares, abren los caminos por los que transitan los campesinos, aplastando y comiendo el sotobosque, que de otra forma es impenetrable y de muy dificultoso transitar. Otro uso fundamental que se le da al Cerro El
Cañi, es para la recolección de piñones, el fruto de la araucaria o pewen (mapudungun). De esta forma, históricamente los habitantes locales suben al cerro durante la época en que el piñon cae de los árboles, empleando para estos los senderos abiertos por las vacas. Bajo esta relación histórica, el lugar era observado por los habitantes locales como un espacio colectivo de uso común. En este sentido, en los relatos de un vecino se señala la tensión que existió entre los habitantes locales y el proyecto de conservación del lugar desde un comienzo:

\footnotetext{
"Ios extranjeros que compraron el cañi eran muy jodidos y que no dejaban recolectar piñones siendo que eran de uno. Nosotros entrabamos animales arriba. Echábamos animales, cerraron al final no pudieron sacar los animales. Los animales viven allá en verano. Los cercos en invierno se rompen. Eso ocurrió varias veces lo de los piñones, pero nunca van a poder prohibir, la gente igual va a buscar piñones, quisieron poner reglas, pero no pudieron" (Jose, sector Pichares).
}

A pesar de que con el tiempo se ha permitido a los vecinos subir al bosque a buscar piñones, el acceso de las vacas sigue siendo un tema de conflicto. Como se dijo anteriormente la relación de los habitantes locales con el Cañi es inalienable de la relación también existente de las vacas con el lugar. Una relación que como se ha dicho tiene raigambre histórica, y vuelve difícil la reconfiguración de estos hábitos. Tal y como señala un vecino al respecto "La gente se crió cortando árboles y criando vacas" (Pedro, sector Pichares). Sin embargo, la crianza de vacas en el contexto económico actual se trata de una práctica de subsistencia. En el momento de realizar el trabajo en terreno eran muy pocos los vecinos, sólo un par, los que seguían subiendo vacas a El Cañi, la mayoría había desistido. De hecho, era común en nuestras conversaciones que se nos hicieran comentarios acerca de los jóvenes y sus trabajos en turismo en la ciudad 
de Pucón, o de los estudios que estos hacían en la vecina ciudad de Temuco o incluso en la capital del país, Santiago. De este modo este lugar se inserta en procesos económicos y territoriales de carácter más global (Gupta y Ferguson, 2008), los cuales en un momento anterior posibilitaban la viabilidad de la cría de ganado, pero que ahora han vuelto difícil la mantención de estas prácticas. Por lo que no ha sido sólo la llegada de los ambientalistas y la creación del área de conservación la que ha dificultado la cría de ganado.

Los campesinos locales entienden la tala de árboles para obtener leña y para abrir terrenos para la cría de ganados como necesarias para mantener el bosque "bajo control". Aquí entran definiciones que observan que domesticar el bosque es fundamental para perpetuar la vida humana en el sector. En una publicación anterior realizada en base a trabajo etnográfico en la misma zona, se observa cómo los significados de la palabra bosque se establecen en oposición a las ideas de selva o monte, las cuales corresponden a las áreas salvajes que se extenderían de manera autónoma a costa de las áreas limpiadas para el trabajo agrícola (ver Digiminiani y Fonck 2015). De este modo, para los campesinos colonos El Cañi formaría parte de lo que es selva o monte. En este sentido, el Cañi en el tiempo ha comenzado a ser visto como un espacio autónomo, que no depende de la intervención humana. De hecho, en un momento histórico el Cañi intentó ser domesticado con el establecimiento de un aserradero. Allí se extraía madera que posteriormente era vendida en otros lugares para realizar productos de carpintería. Al mismo tiempo, debido al frío de la zona, también se usaba la madera de estos árboles para ser quemada y proveer de calefacción. Mientras estábamos en el bosque podíamos observar numerosos cortes realizados por antiguos leñadores en los troncos de árboles centenarios, para ver si estos podían ser útiles para servir como leña o no. También el lugar que ahora es conocido como la laguna seca, lugar donde los visitantes se detienen a observar aves, "fue limpiado" hace muchos años para el pastoreo de ganado. En nuestras conversaciones con campesinos el Cañi siempre era definido como salvaje, quedando cualquier conversación sobre las especies en segundo plano, recalcando más bien los usos que se hacían del lugar. Esto guarda sentido con el contexto colono, dónde la vida se ha construido dominando al bosque, significando el paisaje por medio de la acción humana en el entorno forestal. La manera de concebir la historia local está dada por los significados inscritos en el paisaje por medio de la negociación entre el crecimiento del bosque y la necesidad de mantenerlo bajo control. Valores asignados al paisaje propios de las sociedades colonas (Dominy, 2001; Digiminiani y Fonck, 2015). Los campesinos observan las iniciativas de conservación, impuestas desde voluntades externas, como una amenaza para sus prácticas de subsistencia y de pequeña producción.

\section{5. "Cercas Y Caminos": La Reconfiguración Ambientalista De El Cañi}

Tras la fundación del Santuario El Cañi uno de los propósitos fundamentales de los ambientalistas era reestablecer lo que ellos denominaban los ciclos naturales de la reforestación del bosque. La preocupación principal consistía en proteger y estimular el crecimiento de especies nativas, tales como la Lenga (Nothofagus pumilio), Araucaria (Araucaria araucana), Coihue (Nothofagus dombeyi), las que también son 
valoradas por su condición de endémicas a la zona. Otra razón sostenida por losambientalistas para la valoración positiva del Bosque de El Cañi estaba dada en que muchos de los árboles presentes en el lugar tenían edades muy antiguas, llegando hasta los 800 años en Coihues y Lengas, y 1600 en las araucarias. Esta valoración positiva de las especies endémicas se hace en contraste a la valoración negativa de los bovinos involucrados en las prácticas ganaderas de campesinos locales, y por tanto no fomentadas en el espacio de la reserva Santuario El Cañi. Esta exclusión también se lleva a cabo simbólicamente en los folletos que se hacen del parque, donde se describe detalladamente todas las aves, mamíferos, y plantas que se pueden observar en el santuario. Sin embargo en estas definiciones no se incluye a la vaca, ya que no es considerada como parte del santuario.

Como ya se ha mencionado anteriormente, las vacas han sido una especie problemática para el cumplimiento de los propósitos de conservación. Mientras realizamos recorridos por el bosque con los guías locales, estos nos señalaron la presencia de las vacas que se manifestaba en huellas visibles de su transitar como sus heces, vegetación masticada, y los caminos que abren con sus recorridos. Roberto, guía local de El Cañi, nos mostró cómo la presencia de la vaca como especie que convive en el bosque afecta la presencia o ausencia de otras especies, existiendo mayor biodiversidad cuando las vacas no transitan por la zona. Esta preocupación por la biodiversidad es algo característico de la conservación (Lorimer, 2012). De este modo, donde los bovinos no pastan libremente, hay menos quila, más sotobosque, existiendo también más renovales pequeños de araucarias, lengas y coihues, que no estaban presentes en el espacio no conservado, lo cual era observable a simple vista. Cuando le preguntamos a Roberto por la razón de que esto sucediera, nos comentó que la alimentación de las vacas en base a los renovales, impide la regeneración del bosque buscada por los ambientalistas, quedando sólo árboles más viejos. En una conversación posterior, Roberto nos explicó como los bovinos fomentan también la aparición de la quila, ya que al consumirla favorecen su proliferación y crecimiento mediante sus heces, que esparcen sus semillas. Esta última, por su reproducción densa y rápida, impacta negativamente en la diversidad de las especies que crecen en el bosque, especialmente en el sotobosque. La presencia de Chusquea Quila, de este modo se relaciona negativamente con el crecimiento de otras especies, disminuyendo la biodiversidad.

Una acción relevante en el proceso de conservación en el santuario el Cañi, fue impedir que las especies que viven en él desaparezcan o sean dañadas. De hecho, durante una de nuestras visitas, uno de los guías locales nos comentaba sobre un ambientalista, llamado Charles, que luego de pasar varios días en El Cañi había bajado para descubrir que tenía una rana en su mochila. En vez de dejarla abajo del cerro había vuelto a subir, para dejarla exactamente en el sitio donde él creía que pertenecía, aguantando una dura subida de tres horas sólo para hacer esto. En este tipo de prácticas se puede identificar una consideración y valoración de la naturaleza como algo prístino, algo que Cronon (1996) plantea como constitutivo del ambientalismo de origen norteamericano. En este contexto, los ambientalistas vinculados al Santuario El Cañi entienden las relaciones ecológicas del bosque nativo como frágiles y vulnerables; estableciendo como ideal su 
mantención sin cambios en el tiempo. En este sentido se sostiene el espacio del bosque como un espacio cuya pureza debe ser mantenida y expurgada de influencias externas. El bosque es entendido como un lugar sagrado, argumento relacionado al paradigma de conservación que reproduce la idea de una naturaleza esencial y universal, valorando el bosque por su valor intrínseco, lo que va en consonancia con lo que plantean Ogden y Holmes (2015). Esta esencia se haría presente por medio de la existencia de un frágil equilibrio entre especies que conecta los elementos que habitan al interior del bosque.

Ante esto, el cerco se vuelve un artefacto fundamental para la reconfiguración de las relaciones en el bosque. Al introducir los cercos los guías locales y ambientalistas frenan el avance de las vacas, y por tanto de la Chusquea Quila. En este contexto los guías locales tienen que estar periódicamente reparando las rejas, "llevamos 20 años reparando rejas" (Guía Local). La práctica de reparar los cercos es concebida por los guías no sólo como una tarea de restitución material del cerco, sino una forma de mostrarle a la comunidad local las relaciones con el bosque que el proyecto de conservación busca proponer, manifestándose en estos cercos la idea de un bosque protegido y no sujeto al uso. Al mismo tiempo, esta puede observarse como una práctica de cuidado (Puig de la bellacasa, 2011) mediante la cual los guías locales mantienen un espacio protegido que permita la vida de estas especies de aves, insectos y árboles.

Pero los cercos no siempre funcionan para mantener a las vacas afuera del predio. Esto se da ya sea porque el cerco es aplastado por un árbol de altura que cae en el bosque, o porque los mismos habitantes locales, ante la necesidad de alimentar al ganado, rompen los cercos para dejar pasar a los bovinos al parque. A esto se le suma que los bovinos conocen el territorio, y también presentan habilidades para entrar por su cuenta: "las vacas cachan [saben] cómo llegar al parque" (Guía Local). De hecho, en una las visitas nos encontrábamos descansando en la "laguna seca" junto a los guías locales, y de pronto vimos un montón de vacas aparecer, alimentándose a sus anchas de la tupida vegetación del lugar. En este momento los guías locales se levantaron y fueron a arrear las vacas con varas de quila. Las vacas frente a la intención de los guías, comienzan a moverse en diferentes direcciones para evitar ser atrapadas. Una vez afuera estas no enfilaron hacia abajo del cerro, sino por otros caminos. Mirando el movimiento de las vacas, Roberto nos señaló: "van a volver a entrar, nada que hacer". En este sentido, es posible observar cómo el conflicto entre la comunidad local y el proyecto de conservación no es simplemente un conflicto entre humanos, sino que es una disputa en que otras especies no humanas, como las vacas, juegan un rol activo, y que esto se da por las relaciones específicas de mutua afectación que estas establecen con las otras especies del lugar.

\section{6. "El Bosque Llama": Cultivando La Capacidad De Cuidar EI Bosque}

La propuesta socio-ecológica de los ambientalistas para el Santuario El Cañi se sostiene sobre concepciones del bosque nativo asociadas a los valores de la ecología profunda. La ecología profunda es una postura políticofilosófica desarrollada por el filósofo noruego Arne Naess, caracterizada por proponer una visión holística ecocéntrica de simetría en la 
relación entre el humano y el medio ambiente (Naess, 1973). En las actitudes hacia el bosque de los ambientalistas existe una combinación de propósitos espirituales y preocupaciones científicas sobre la conservación. Para Rod - ambientalista británico vinculado estrechamente al proyecto de conservación y la educación ambiental -el bosque es un sitio revelador, que puede estimular nuevas formas de relación y conciencia ambiental entre los visitantes, tal como invita en su manuscrito pewen inspirado en el Cañi. A partir de esta enseñanza, Rod mencionaba constantemente la necesidad de "caminar por el bosque con los sentidos abiertos", para poder recibir los mensajes y estar atentos a las presencias que el Cañi aporta al caminante. Para los guías de El Cañi, científicos y ambientalistas extranjeros estar en el bosque es una experiencia multisensorial, y no refiere meramente al contacto con la flora nativa, sino que también hace referencia a la red de relaciones que se configuran en el bosque, las cuales incluyen a los animales, tales como pájaros o insectos, que forman parte de él. De este modo cuando los guías dicen que el "bosque llama", no se refieren a una especie de nuevo animismo (Descola, 2013) en que los árboles llamarían a los paseantes, sino que más bien se refieren a la red de relaciones, que se manifiestan de modo sonoro, con los sonidos característicos que emiten las distintas especies y que llaman a contemplarlas y a entrar en relación con ellas. El caminar por el bosque genera que las trayectorias humanas se crucen temporal y espacialmente con las trayectorias de seres no humanos, las cuales son puestas en evidencia por los distintos cantos o ruidos que emiten las distintas especies. De este modo el Ilamado del bosque se vuelve una invitación auditiva, que se aparece ante el paseante al estar inmerso en un paisaje sonoro o soundscape siguiendo la aproximación de Murray Schafer (1977).

Parailustrarestaidea, cabenarrarunaexperiencia ocurrida subiendo hacia El Cañi por Pichares, sendero oficial de entrada al parque. Después de pasar el primer refugio nos vimos sumidos entre los Coihues y las Lengas, que conforman la primera parte del bosque que se manifiesta al visitante, ya que estas son especies que habitan en menor altura que la araucaria. Mientras caminábamos, el guía local Roberto, quién caminaba silenciosa y contemplativamente, nos iba señalando las distintas especies con las que nos íbamos encontrando entre los árboles. En más de una ocasión, nosotros pasamos de largo, sin percibir lo que él podía detectar, teniendo que retroceder ante lo que él nos señalaba. En una de estas instancias nos hizo detenernos a mitad de camino, y nos señaló lo que parecía ser el tronco rugoso de un árbol cubierto de musgo. De pronto oímos un aleteo y vemos que había una especie de escarabajo herido, la cual Roberto toma con delicadeza entre sus manos, enseñándonos sus partes y sus alas. Fue mucha la sorpresa para todos pues no habíamos podido distinguirlo entre el sotobosque hasta que él lo señaló.

En todo momento durante nuestra caminata, Roberto nos instaba a desplazarnos en silencio, porque de otro modo y con una actitud diferente espantaríamos a las aves que habitaban el bosque que son de por sí muy sensibles al ruido. De este modo, caminamos en silencio, escuchando de vez en cuando los llamados de las aves, los cuales se cruzaban en nuestra trayectoria por el bosque. Con esto nos fuimos dando cuenta de que las aves son mucho más fáciles de oír que de observar, y que al movernos silenciosamente era posible escuchar 
los "Ilamados" que hacían con sus cantos. Nos ocurrió en más de una ocasión que Roberto escuchaba las aves y podía detectar dónde estaban, pero al observar nosotros el lugar señalado por Roberto, éramos incapaces de ver sus siluetas, observando en cambio solo ramas de quila. Esto pasó con diversas aves (Fíofío, Carpinterito, Carpintero gigante, Chucao, Chercán, Rayadito, Picaflor, Hued-hued, Churrin, Colilarga, Cometocinos, Churrin, Zorzal, Canquén entre otras), que eran indistinguibles para nosotros y se perdían entre la vida vegetal, hasta que de pronto ya nos aproximamos al sector del bosque donde habitan las araucarias. Fue en este momento que oímos un golpeteo sobre nuestras cabezas, un ruido seco pero leve, como de un pequeño martillo. En este momento Roberto nos señala un árbol seco a unos metros de nosotros, subimos la mirada y de pronto vemos un carpintero golpeteando el árbol. A diferencia de otros pájaros con colores más miméticos, la cabeza roja del carpintero en contraste con el tronco opaco reveló su presencia enseguida, afectandonos desde su corporalidad. Unos metros más allá, después de la desaparición del carpintero, Roberto se acerca a otro tronco, también seco próximo al camino y nos señaló unos agujeros que hay en él. Nos cuenta que estos son de carpintero, diciendo que esta ave se come los gusanos de la madera podrida. A partir de este momento, gracias a las observaciones de Roberto, empezamos a distinguir las huellas de esta ave y sus cantos en el bosque. sus procesos de vida, que para nuestra sorpresa, estaban por doquier en el camino que acabamos de recorrer. En este sentido, una persona no puede escuchar el llamado del bosque de buenas a primeras, sino que más bien debe entrenarse, debe desarrollar una sensibilidad para poder hacer esto. Debe aprender a "caminar con los sentidos abiertos" y observar lo que el bosque va mostrando, dejándose afectar (Massumi, 2015) por estos seres no humanos que habitan el bosque.

Profundizamos en esta perspectiva durante una conversación que tuvimos con Alejandro, guardaparques en formación, quién se encontraba haciendo su práctica profesional en el Santuario El Cañi. Mientras compartíamos un mate el comentaba que la primera vez que subió al parque no vió nada, que se limitó a subir y a bajar lo más rápido posible. Hizo el recorrido del parque, hasta el mirador, en unas cuatro horas, ida y vuelta. Cuando llegó abajo Manuel y Roberto primero se sorprendieron, y luego lo reprendieron, “¿Para qué subiste?” señalándole que lo realizado no era inútil ya que no había podido experimentar el bosque. Alejandro se dió cuenta de que estos tenían razón, que no recordaba nada de lo que había visto en su travesía, que sólo se había guiado por el deseo de finalizar el trayecto, como si fuera una tarea a cumplir. Al día siguiente subió de nuevo y se quedó tres días arriba. "ahí pude entender más lo que pasaba" nos confesó. A medida que la conversación seguía, Alejandro nos fue develando como el bosque, y el poder conocer este bosque, no sólo se componía de un conocimiento de sus especies, sino también de saber andar por sus caminos, por medio de una actitud. Según él, este conocimiento sólo se puede adquirir caminando por el bosque, una y otra vez. Esto es algo que logramos corroborar en nuestra expediciones al lugar, dónde caímos en cuenta de que el andar por los caminos era fundamental para la interacción de nosotros como humanos con las otras especies del bosque, entrando en una relación afectiva que estaba mediada por el conocimiento adquirido a través de nuestro propio movimiento a través del lugar (Ingold, 2000). 
Al recorrer una y otra vez los caminos de El Cañi, empieza a aparecer una recurrencia, los árboles adquieren una historicidad en relación a nosotros como humanos y se generan configuraciones de especies que van marcando el camino de los guías y de nosotros mismos como visitantes del bosque. Siguiendo nuestra propia vivencia en recorridos por el bosque, las especies se comenzaron a individualizar, afectándonos desde su singularidad. Así, un coihue dónde vimos una especie particular comenzaba a adquirir un sentido único, volviéndose reconocible al caminar. De hecho, como parte del trabajo de campo en el Santuario, compartimos la experiencia del bosque, en la zona más alta donde habitan las araucarias, con un equipo de ecólogos especializados en ornitología y conservación, midiendo la presencia de aves y los hábitos de nidificación de las aves en el Cañi. En un momento dejamos una escalera bajo una lenga muerta en pie, y a la vuelta del día de trabajo pasamos a buscarla. Cuando llegamos al árbol, varias horas después, de pronto oímos un sonido sobre nuestras cabezas. Era un cría de carpintero vocalizando asomada desde una cavidad en la zona alta de la Lenga. Los ornitólogos trabajan en general sobre hábitos de nidificación, midiendo, localizando y siguiendo durante las estaciones del año los nidos encontrados. Encontrar un nido nuevo, caminando por el bosque es un hallazgo poco usual e inspirador. Fue un momento de afectación y maravilla para todos nosotros, pues lo observamos como un regalo del bosque y del carpintero, que estaba allí esperándonos y observandonos desde la altura. Luego, al proseguir nuestro trabajo en terreno y pasar por ahí recordábamos ese momento particular como una situación de encuentro afectivo con el bosque. Quedando marcada la historia de esa Lenga con nosotros, y de ese pequeño carpintero curioso desde su nido en aquel árbol. De esta forma comprendimos que la topografía de El Cañi se encuentra llena de capas de significados (Basso, 1996), los cuales se van configurando en relación a este tipo de encuentros afectivos.

\section{Discutiendo "El Llamado Del Bosque"}

Revisando etnográficamente las prácticas de vida en el "Santuario el Cañi", es posible apreciar cómo en un mismo espacio de conservación se encuentran presentes diferentes perspectivas humanas; las campesinas y las ambientalistas, las cuales han entrado en tensión y colaboración durante los últimos 20 años en el caso estudiado. En este sentido, se puede plantear que la conservación se encuentra estrechamente relacionada a valores y apreciaciones de la así llamada "naturaleza" (Jax y Rozzi, 2004; Cheng et al., 2003). De hecho el tipo de relaciones que generan los ambientalistas, campesinos y visitantes con el bosque podrían ser observadas como lo que Low describe como "place attachment", relaciones simbólicas y afectivas que humanos generan con un lugar, mediante emociones que son compartidas culturalmente (1992:165). Esta visión es compatible también con la idea de "placemaking" de Schneekloth \& Shibley (1995), quienes describen las formas simbólicas en que los espacios son vividos por grupos humanos, ayudan a dar sentidos compartidos a un espacio y afectan la comprensión de este. En este sentido, siguiendo la perspectiva centrada en los humanos criticada en el presente artículo, las tensiones entre ambientalistas y la comunidad local pueden ser observadas como un conflicto entre significados, o relaciones simbólicas en disputa. A pesar de esto, nuestra 
experiencia etnográfica nos enseñó que esto no es suficiente para comprender la situación vivida en El Cañi. Esto debido al hecho de que los seres humanos no eran los únicos compartiendo y habitando en ese espacio, sino que formaban parte de una red de relaciones en la que los no humanos son participantes activos (Ingold, 1995; Haraway, 2008). De este modo se vuelve relevante no sólo la relación entre ambientalistas, que buscan promover la conservación de un espacio, y campesinos, que buscan perpetuar el uso tradicional de ese mismo, sino también las diversas relaciones de afecto que ambientalistas y campesinos poseen con las diferentes especies que habitan el Cañi. Se debe resaltar que las vacas permanecen como una especie problemática dentro de estas relaciones. En este sentido la reconfiguración que ocasiona la irrupción del proyecto ambiental no reconoce la relación de vacas y campesinos como especies compañeras (Haraway, 2008), quedando estas sin un lugar en El Santuario El Cañi y generando un desafío que perdura en el tiempo. Esta contradicción se desvela desde el enfoque en las relaciones interespecies, sacando a la luz controversias y exclusiones de los procesos propios de la conservación ambiental.

Sin embargo, por otro lado el caso de estudio nos enseña la importancia de oír "el llamado del bosque" y la capacidad de escucharlo, desarrollando un actitud de cuidado y reconocimiento de las vitalidades del bosque. Para oír el llamado del bosque es necesaria la apertura hacia estos otros seres no humanos que habitan en conjunto con los humanos en ese espacio. En algún sentido, "el llamado del bosque" es un sentient concept (Simonetti, 2017), un concepto que nace de la involucración corporal y de las prácticas in situ de campesinos, guías locales, científicos, ambientalistas y de nosotros mismos como paseantes. Para esto se debe ir un poco más allá, comprendiendo que "oír el llamado del bosque" es también encontrarse abierto a dejarse afectar por los seres no humanos que conforman este bosque. Entendiendo afecto en la acepción de Spinoza "afectar y ser afectado" que rescatan Massumi (2015) y Gregg \& Seigworth (2009). Pero este afectarse, en el caso de los ambientalistas, guías locales y científicos, ha conducido a lo que Maria Puig de la Bellacasa (2011) ha llamado cuidado, generando un compromiso ético hacia el cuidado de estos seres no humanos entre las personas que pueden oír su llamado. Al ser capaces de "oír el llamado del bosque" estas personas empiezan a discernir y estar comprometidas éticamente con las distintas formas de vida que guarda el bosque. Lo que guarda relación con lo que la filósofa Jane Bennet ha expresado como: "La tarea ética a la mano aquí es la de cultivar la habilidad de discernir la vitalidad no humana, de volverse perceptualmente abiertos a ella" (2010:14). Se debe dar cuenta también, que esta es una forma muy particular del cuidado, pues posee el riesgo de establecer relaciones asimétrica con la naturaleza y especies, en que estas son tratadas como entidades frágiles que deben ser protegidas.

Es importante dar cuenta de que el establecer relaciones afectivas y éticas con la naturaleza, capaces de reconocer las relaciones entre especies no es una novedad traída exógenamente por los ambientalistas a la zona de El Cañi. Históricamente, desde el conocimiento mapuche se ha dado cuenta de las múltiples vidas que se entrelazan en la tierra "un cúmulo de alteridades que están permanentemente llamadas a interactuar" (Pichinao, 2015: 98), entre las cuales se 
encuentran árboles, ríos, montañas, árboles, insectos, siendo los seres humanos uno más. En este sentido, no se debe considerar que la relación afectiva de cuidado con las especies es algo novedoso de los discursos y prácticas ambientalistas externos a El Cañi, sino que es un tipo de relación que ya estaba en las prácticas locales, pero opacada por las lógicas extractivistas que históricamente ha favorecido el estado chileno (Skewes et al., 2011; Pichinao, 2015). De todas formas, se debe esclarecer que la relación que mantienen los pueblos indígenas con otras especies no funcionan con las mismas lógicas que el ambientalismo americano, que establece una naturaleza prístina, pura y frágil, que debe ser cuidada y protegida. Por ejemplo, en las prácticas de diversos grupos indígenas es posible notar la coexistencia de relaciones de cuidado, con prácticas de cacería y consumo de otras especies (Goldman et al., 2011).

Como plantea Campbell (2005) es la idea de naturaleza proveniente del paradigma de la conservación la que puede ser problemática, ya que corre el riesgo de concebir a ésta como algo estático, sin cambios, presentando dificultades para abordar las interacciones que ocurren dentro de ella. En esta línea se ha afirmado, que el tipo de conservación de vida silvestre de estilo norteamericano, no es viable para áreas rodeadas con comunidades cuyos estilos de vida se encuentran imbricados con la biodiversidad (Stevens, 1997)2. A pesar de que el Santuario El Cañi viene a cortar ciertas relaciones socio-ecológicas en la zona, imponiendo otras bajo un contexto histórico neoliberal global, este sí ha sido exitoso al momento de generar relaciones de colaboración afectivas con el bosque, representadas principalmente

Para el caso Chileno ver Observatorio Ciudadano (2016) en la actitud de los guías. De este modo ha acontecido una labor educativa sintiente, cuya base es la apertura hacia las especies del bosque, y que desemboca en una forma particular de cuidado hacia estas. Cabe señalar también que el proyecto de conservación se ha desarrollado bajo un contexto de migración campo-ciudad relacionado a la falta de empleo local y a la necesidad de generar ingresos monetarios (Vergara, 2006), lo que ha llevado a que las prácticas de subsistencia tradicionales de los campesinos de la zona, entre las que se encuentra la ganadería, de a poco han ido disminuyendo. En este sentido, si bien se deja fuera prácticas campesinas anteriores, este sistema de producción se ha visto alterado por un contexto histórico, ambiental y económico más amplio.

\section{Conclusión}

Con este trabajo se busca ir más allá de la dicotomía entre comunidad local y ambientalista, como lo han hecho los trabajos clásicos sobre conservación y sociedad que sitúan esta disputa como algo que ocurre sólo entre humanos. Siguiendo el caso del Cañi desde una experiencia etnográfica, en la práctica, se ha observado como dentro de las relaciones entre proyecto de conservación y comunidad local tienen relevancia también los vínculos entre especies. Por un lado se revelaron los conflictos entre las vacas, especie compañera de los campesinos, y las prácticas de conservación ambiental instaladas en el Santuario El Cañi. Por otro lado se destacaron las relaciones de afecto existentes entre los guías locales, ambientalistas y distintas especies de árboles, pájaros e insectos, observando como estas formas situadas de 
afecto surgen a raíz de encuentro corporales en el bosque. Estos conflictos, y también esta afectividad y sensibilidad hacia el bosque, no pueden ser concebidas simplemente desde la disputa entre prácticas locales y discursos globales (Tsing, 2010). Siguiendo el Ilamado de Escobar, los modelos locales de naturaleza no pueden ser explicados sin referencia a lugares, fronteras y culturas locales concretas. Retienen una especificidad del lugar. Deben ser recontextualizados con otros regímenes de naturaleza y las fuerzas globales en los que se encuentran inmersos (Escobar, 1999).

El enfoque presentado permite discutir los desafíos de la conservación con respecto a las comunidades locales desde la especificidad del Cañi como lugar y desde las naturalezas que están en juego, bajo las constelaciones de prácticas interespecies que acontecen en estos bosques del sur de Chile. Finalmente, este caso nos invita a reflexionar sobre la conservación y el cuidado desde una actitud basada en lo afectivo y en el reconocimiento de la presencia y mutua afectación entre los seres que habitan el Cañi. Esto permitiría, desde una perspectiva teórica, realizar una redefinición de lo que ha sido considerado como comunidad local por los estudios de la conservación, ampliando su definición al incluir a los seres no humanos que se encuentran participando activamente en los complejos desafíos de la conservación.

\section{Bibliografía}

Basso, K. 1996. Wisdom sits in places: landscape and language among the western Apalache. Albuquerque, NM: University of New Mexico Press.

Bennet, J. 2010. Vibrant matter: a political ecology of things. Duke University Press.

Bengoa, J. 2002. Historia de un conflicto el estado y los mapuches en el siglo XX. Santiago, Chile: Editorial Planeta.

Brockington, D. 2002. Fortress conservation: the preservation of the Mkomazi game reserve. Tanzania. London. The International African Institute.

Brosius, J. P., Tsing, A. L., y Zerner, C. 1998. "Representing communities: Histories and politics of community-based natural resource management." Society \& Natural Resources 11(2): 157168.

Campbell, B. 2005. "Changing protection policies and ethnographies of environmental engagement." Conservation and Society 3(2): 280.

Camus, P. y Lazo, A. 2014. Guardaparques, su historia y vivencias en la contribución a la conservación del Sistema Nacional de Áreas Silvestres Protegidas del Estado. CONAF y Proyecto MMA/GEF/PNUD Creación de un Sistema Nacional Integral de Áreas Protegidas para Chile: Estructura Financiera y Operacional (proyecto GEF-SANAP).

Catalán, E. 2015. Relaciones humano-ambiente en el Parque Nacional La Campana: Una trayectoria de encuentros y desencuentros entre Comunidades Locales y el Área Protegida
(Tesis para el grado de Antropóloga Social, U. Chile).

Catalán, R., P. Wilken, A. Kandzior, D. Tecklin, H. Burschel. 2006. Bosques y comunidades del sur de Chile. Santiago, Chile: Universitaria.

Cheng, A.S., Kruger, L.E., Daniels, S.E., 2003. "'Place" as an integrating concept in natural resource politics: propositions for a social science research agenda." Soc.Nat. Resour. 16 (2), 87-104.

Cronon, W. 1996. Uncommon ground: Rethinking the human place in nature. Nueva York: W. W. Norton y Company

Crutzen, P.J. (2002) "Geology of mankind." Nature, 415(3): 23. Descola, P. 2013. Beyond Nature and Culture. Chicago: Chicago University Press.

Digiminiani P. y M. Fonck, 2015. "El paisaje como proceso de vida." Revista del Norte Grande 61:7-24

Dominy, M. 2001. Calling the Station Home: Place and Identity in New Zealand's High Country. Lanham: Rowman and Littlefield.

Goldman, M. Nadasdy, P. y Turner, M. 2011. "Knowing Nature: Conversations at the Intersection of Political Ecology and Science Studies". Chicago: University of Chicago Press.

Gupta, A., J. Ferguson. 2008. "Más allá de la "cultura": Espacio, identidad y las políticas de la diferencia." Antípoda $\mathrm{n}^{\circ} 7$ julio-diciembre de 2008, pp. 233-256.

Gregg, M., G. Seigworth. 2009. The Affect Theory Reader. Durham \& London, UK: Duke University Press.

Escobar, A. 1999. "El mundo postnatural: elementos para 
una ecología política anti-esencialista." En El Final del salvaje: antropologia, cultura y politica en la antropología contemporánea. Bogota: Cerec.

Evans, J., y Jones, P. 2011. "The walking interview: Methodology, mobility and place." Applied Geography, 31(2): 849858.

Haraway, D. 2008. When Species Meet. Minneapolis: Univ Of Minnesota Pres

Hidalgo, R. and H.M. Zunino. 2011. Negocios inmobiliarios en centros turísticos de montaña y nuevos modos de vida: el papel de los migrantes de amenidad existenciales en la Comuna de Pucón - Chile. Estudios y Perspectivas en Turismo 20: 307326

Ibarra, J.T., N. Gálvez, A. Gimona, T. Altamirano, I. Rojas, A. Hester, J. Laker and C. Bonacic. 2012. "Rufous-legged Owl (Strix ru pes) and Austral Pygmy Owl (Glaucidium nanum) stand use in a gradient of disrupted and old growth Andean temperate forests, Chile." Studies on Neotropical Fauna and Environment 47: 33-4

Ingold, T, 1995 "Building, dwelling, living: how animals and people make themselves at home in the world," in Shifting Contexts: transformations in anthropological knowledge (ed M Strathern), 57-80, Routledge, London.

Ingold, T. 2000. The Perception of the Environment. London, Uk: Routledge

Jax, K., R. Rozzi. 2004. "Teoría ecológica y valores en la definición de objetivos de conservación: ejemplos de regiones templadas de Alemania, Estados Unidos de América y Chile." Revista Chilena de Historia Natural 77: 349-366.

Jones C. 2012. "Ecophilantropy, neoliberal conservation, and the transformation of Chilean Patagonia's Chacabuco Valley." Oceania 82(3): 250-263.

Jorquera-Jaramillo C., J.M. Alonso', J. Aburto, K. MartínezTillería, M.F. Leon, M.A. Pérez C.F. Gaymer, F.A. Squeo. 2012. "Conservación de la biodiversidad en Chile: Nuevos desafíos y oportunidades en ecosistemas terrestres y marinos costeros." Revista Chilena de Historia Natural 85: 267-280.

Kirksey, E. y Helmreich, S. 2010. "The emergence of multiespecies ethnography." Cultural Anthropology 25 (4): 545-576.

Kusenbach, M. 2003. "Street Phenomenology. The Go-Along as Ethnographic Tool.” Ethnography 4: 455-85.

Kohn, E. 2013. How Forests Think: Toward an Anthropology Beyond the Human. Berkeley: University of California Press.

Klubock, T. 2014. La Frontera: Forests and Ecological Conflict in Chile's Frontier Territory. Durham: Duke University Press.

Latimer, J., M. Miele. 2013. "Naturecultures? Science, Affect and the Non-human" en Theory, Culture \& Society Volume 30 Numbers $7 / 8$.

Latour, B. 2004. Politics of Nature: How to Bring the Sciences into Democracy. (C. Porter, Trans.). Cambridge, Mass: Harvard University Press.

Low, S., I. Altman. 1992. Place attachment. Springer: New York London

Lorimer, J. 2012. "Multinatural geographies for the
Anthropocene." Progress in Human Geography 36(5) 593-612. Massumi, B. 2015. Politics of Affect. Cambridge, UK: Polity.

Murray Schafer, 1977. The soundscape: our sonic environment and the tuning of the world. Rochester: Destiny Books.

Navaro-Yashin, Y. 2012. The Make-Believe Space: Affective Geography in a Post-War Polity. Durham: Duke University Press.

Naess, Arne. 1973. "The Shallow and the Deep, Long-Range Ecology Movement: A Summary." Inquiry: An Interdisciplinary Journal of Philosophy and the Social Sciences 16: 95-100.

Observatorio Ciudadano (2016). Cuestionando los enfoques clásicos de conservación en chile. El aporte de los pueblos indígenas y las comunidades locales a la protección de la biodiversidad. Temuco: Observatorio Ciudadano, Consorcio TICCA y IWGIA.

Ogden, L., G. Holmes. 2015. "Involucramientos globales del bosque, incluso en el fin del mundo." En B. Bustos, M. Prieto y J. Barthon (eds.). Ecología política en chile, naturaleza, sociedad, conocimiento y poder. Santiago de Chile: Editorial Universitaria.

Petitpas, R., Ibarra J. T., M. Miranda, and Bonacic C. 2016 "Spatial patterns over a 24-year period show an increase in native vegetation cover and decreased fragmentation in Andean temperate landscapes, Chile." Ciencia e investigación agraria 43(3):384-395

Pichinao, J. 2015. "La mercantilización del mapuche mapu:hacia la expoliación absoluta". Awükan ka kuxankan zugu Wajmapu mew: Violencias coloniales en Wajmapu. Antileo, E., Cárcamo-Huechante, L., Calfío, M., Huinca-Piutrin, H. (eds.). Santiago, Chile: Ediciones Comunidad de Historia Mapuche.

Puig de la Bellacasa, M. 2011. "Matters of care in technoscience: Assembling neglected things." Social Studies of Science 41(1) 85-106

Robbins, P. 2004. "Capitulo 1: Political versus Apolitical Ecologies." Political ecology: A critical introduction. Malden MA Blackwell.John Wiley \& Sons. (p. 11-24).

Schneekloth, L., R. Shibley. 1995. Placemaking: The Art and Practice of building communities. New York Chichester Brisbane Toronto: John Wiley and sons.

Scott, G. 2002. The Cañi Reserve. Stanford Graduate School of Business.

Simonetti, C. (2017). Sentient Conceptualisations: Feeling for Time in the Sciences of the Past (1 edition). Abingdon, Oxon; New York, NY: Routledge.

Skewes, J., D. Guerra, P. Rojas, M.A. Mellado. 2011. "¿La memoria de los paisajes o los paisajes de la memoria? Los enigmas de la sustentabilidad socioambiental en las geografías en disputa." Desenvolvimento e Meio Ambiente, n. 23, p. 39-57.

Stengers, I. 2010. "Including Nonhumans in Political Theory: Opening Pandora's Box?" En Political Matter: Technoscience, Democracy, and Public Life B. Braun \& S. Whatmore (eds). Minneapolis, USA: University of Minessota Press. 
Stevens, S. (ed). 1997. Conservation through survival: indigenous peoples and protected areas. Washington, DC: Island Press.

Tsing, A. 2010. Adat/Indigenous: Indigeneity in Motion. En A Tsing \& C Gluck (eds), Words in Motion. Duke University Press, Durham, pp. 40-66.

Tsing, A. L. 2012. "Unruly Edges: Mushrooms as companion species for Donna Haraway." Environmental Humanities (1):141-154.
Vargas, A., D. Díaz. 2014. "Community-based conservation programs and local people willingness to pay for wildlife protection: The case of the cotton-top tamarin in the Colombian Caribbean." Lecturas de Economía - No. 81. Medellín, Colombia.

Vergara, C. 2006. "Utilización de recursos forestales en dos comunidades campesinas de la provincia de Valdivia." En Bosques y comunidades del sur de Chile. Catalán, R., P. Wilken, A. Kandzior, D. Tecklin, H. Burschel (eds.). Santiago, Chile: Universitaria 